This item was submitted to Loughborough's Research Repository by the author.

Items in Figshare are protected by copyright, with all rights reserved, unless otherwise indicated.

\title{
On enabling mobile crowd sensing for data collection in smart agriculture: a vision
}

\section{PLEASE CITE THE PUBLISHED VERSION}

https://doi.org/10.1109/JSYST.2021.3104107

\section{PUBLISHER}

Institute of Electrical and Electronics Engineers

\section{VERSION}

AM (Accepted Manuscript)

\section{PUBLISHER STATEMENT}

(c) 2021 IEEE. Personal use of this material is permitted. Permission from IEEE must be obtained for all other uses, in any current or future media, including reprinting/republishing this material for advertising or promotional purposes, creating new collective works, for resale or redistribution to servers or lists, or reuse of any copyrighted component of this work in other works.

\section{LICENCE}

\section{All Rights Reserved}

\section{REPOSITORY RECORD}

Sun, Yuanhao, Weimin Ding, Lei Shu, Kailiang Li, Eve Zhang, Zhangbing Zhou, and Guangjie Han. 2021. "On Enabling Mobile Crowd Sensing for Data Collection in Smart Agriculture: A Vision”. Loughborough University. https://hdl.handle.net/2134/16885351.v1. 


\title{
On Enabling Mobile Crowd Sensing for Data Collection in Smart Agriculture- A Vision
}

\author{
Yuanhao Sun, Weimin Ding ${ }^{\star}$, Lei Shu^, Edmond Nurellari, Kailiang Li, Yu Zhang, Zhangbing Zhou, and \\ Guangjie Han
}

\begin{abstract}
Smart agriculture enables the efficiency and intelligence of production in physical farm management. Though promising, due to the limitation of the existing data collection methods, it still encounters few challenges that are required to be considered. Mobile Crowd Sensing (MCS) embeds three beneficial characteristics: a) cost-effectiveness, b) scalability, and c) mobility and robustness. With the Internet of Things (IoT) becoming a reality, the smart phones are widely becoming available even in remote areas. Hence, both the MCSs characteristics and the plug and play widely available infrastructure provides huge opportunities for the MCS-enabled smart agriculture.opening up several new opportunities at the application level. In this paper, we extensively evaluate the Agriculture Mobile Crowd Sensing (AMCS) and provide insights for agricultural data collection schemes. In addition, we provide a comparative study with the existing agriculture data collection solutions and conclude that AMCS has significant benefits in terms of flexibility, collecting implicit data, and low cost requirements. However, we note that AMCSs may still posses limitations in regard to data integrity and quality to be considered as a future work. To this end, we perform a detailed analysis of the challenges and opportunities that concerns the MCS-enabled agriculture by putting forward six potential applications of AMCS-enabled agriculture. Finally, we propose future research and focus on agricultural characteristics, e.g., seasonality and regionality.
\end{abstract}

Index Terms-Mobile crowd sensing, smart agriculture, data collection, Internet of Things

\section{INTRODUCTION}

Big Data technology combines the mathematical models in the smart agriculture domain to seamlessly analyse a large amount of data in agricultural production and provide valuable insights to the farmer without the need of the dedicated specialist. It is one of the most promising approach to solve the long-standing challenges [1], e.g., unmarketable agricultural products due to lack of information exchange between farmers and consumers. Currently, two main approaches have been widely applied in agricultural data acquisition: a) Site survey with dedicated professionals [2]; b) Sensing technology based on Space-Air-Ground Integrated Network (SAGIN) [3]. Nevertheless, both the aforementioned approaches are not feasible and scalable in the current and future agriculture paradigm. Just to mentioned few of them, e.g., the site survey approach is not suitable for Big Data technology because it is time-consuming, labour-intensive, and only samples smallscale local data/information. On the other hand, the SAGIN approach has several limitations, e.g., very high deployment and operational cost. In addition, it does not allow further

* Weimin Ding and Lei Shu are corresponding authors improvements in terms of the flexibility and scalability of deployed devices, which is clearly a bottleneck and highly desirable in the context of Agriculture. Thus, this work provides a platform that evaluates and proposes new data collection approaches to overcome the aforementioned limitations.

Mobile Crowd Sensing (MCS) is a technique where a large number of individuals having mobile devices, e.g., smart phones and wearable equipment, capable of sensing and sharing information of interest, aiming to complete large-scale and complex sensing tasks with three beneficial characteristics: 1) cost-effectiveness, 2) scalability and 3) mobility [4] - [7]. Recently, there has been a growing interest on research based on the existing developed systems that proposes different approaches to improve the availability of MCS technology for different application scenarios. The existing typical applications of MCS can be divided into seven parts, as shown in Table I.

- Environmental Monitoring. Sensing environmental information is fundamental for sustainable urban development and can improve citizens' quality of life. Aiming to monitor air quality, in [8], authors proposed a framework, namely CrowdRecruiter, to minimize incentive payments by selecting a small number of participants while still satisfying probabilistic coverage constraint. GRC-Sensing [10] and Urban Safety [11] were utilized to monitor noise pollution and information of damaged urban infrastructure, respectively. In [24], the authors first built the SenSquare system to embrace data availability and device heterogeneity. Then, they constantly improved the system's function, e.g., classifying heterogeneous data [25] and designing an easy visual programming plugin [26].

- Living Service. This category can help citizens get a better life experience. In [9], CrowdQTE was presented to utilize the sensor-enhanced mobile devices and crowd human intelligence to monitor and provide real-time queue time information for various queuing scenarios. Authors designed Mobibee to realize indoor localization by inspiring citizens to contribute their data [19] and proposed a truth discovery algorithm to detect falsified data [20]. Besides, there are also other services, e.g., recycling waste with WasteApp [22] and measuring wireless performance with MCNet [23].

- Disaster Prediction. Large magnitude earthquakes can continue to kill and injure a large of people, inflicting lasting societal and economic disasters. The prediction 
TABLE I

TYPICAL APPLICATIONS OF MCS

\begin{tabular}{|c|c|c|c|c|c|}
\hline Developed system & Functional description & Used sensors & Identity of participant & Research direction & Application area \\
\hline CrowdRecruiter [8] & Monitoring air quality & GPS, air quality sensor & Citizens & Participant selection & Smart environment \\
\hline CrowdQTE [9] & Predicting queue time & GPS, microphone & Citizens & Function realization & Smart service \\
\hline GRC-Sensing [10] & Monitoring noise pollution & $\begin{array}{l}\text { GPS, accelerometer, } \\
\text { microphone }\end{array}$ & Citizens & Function realization & Smart environment \\
\hline Urban Safety [11] & $\begin{array}{l}\text { Monitoring urban infras- } \\
\text { tructure }\end{array}$ & $\begin{array}{l}\text { GPS, accelerometer, } \\
\text { video camera }\end{array}$ & Citizens & Function realization & Smart service \\
\hline CREAM [12] & Predicting traffic conditions & GPS, video, camera & Citizens & Function realization & Smart transportation \\
\hline TrackYourTinnitus [13] [14] & $\begin{array}{l}\text { Collecting tinnitus informa- } \\
\text { tion }\end{array}$ & $\begin{array}{l}\text { GPS, accelerometer, } \\
\text { microphone }\end{array}$ & Patients & $\begin{array}{l}\text { Function realization; } \\
\text { data mining }\end{array}$ & Smart health \\
\hline MyShake [15] [16] & Earthquake early-warning & GPS, accelerometer & Citizens & Function realization & Smart service \\
\hline TrackMaison [17] [18] & $\begin{array}{l}\text { Improving network service } \\
\text { quality }\end{array}$ & $\begin{array}{l}\text { GPS, accelerometer, } \\
\text { microphone, camera }\end{array}$ & Netizens & $\begin{array}{l}\text { Data analysis and } \\
\text { visualization }\end{array}$ & Smart social \\
\hline MobiBee [19] [20] & Indoor localization & GPS, camera, bluetooth & Citizens & $\begin{array}{l}\text { Incentive mechanism; } \\
\text { data authenticity }\end{array}$ & Smart service \\
\hline SafeStreet [21] & Road anomaly detection & $\begin{array}{l}\text { GPS, accelerometer, } \\
\text { camera }\end{array}$ & Citizens & Function realization & Smart transportation \\
\hline WasteApp [22] & Recycling waste & GPS, camera & Citizens & Function realization & Smart service \\
\hline MCNet [23] & $\begin{array}{l}\text { Measuring wireless perfor- } \\
\text { mance }\end{array}$ & $\begin{array}{l}\text { GPS, signal receiver, } \\
\text { communication module }\end{array}$ & Citizens & $\begin{array}{l}\text { Function realization; } \\
\text { incentive mechanism }\end{array}$ & Smart service \\
\hline SenSquare [24] [25] [26] & Monitoring environment & $\begin{array}{l}\text { GPS, microphone, } \\
\text { Wi-Fi, timer }\end{array}$ & Citizens & Data processing & Smart environment \\
\hline CrowdOS [27] & Application integration & $\begin{array}{l}\text { GPS, gravity sensor, am- } \\
\text { bient light sensor, gyro- } \\
\text { scope, proximity sensor }\end{array}$ & Citizens & $\begin{array}{l}\text { Task allocation; } \\
\text { data processing }\end{array}$ & $\begin{array}{l}\text { Smart environment } \\
\text { and service }\end{array}$ \\
\hline CovidSens [28] & $\begin{array}{l}\text { Monitoring the COVID-19 } \\
\text { propagation }\end{array}$ & $\begin{array}{l}\text { GPS, microphone, } \\
\text { camera }\end{array}$ & All & $\begin{array}{l}\text { Function realization } \\
\text { privacy protection }\end{array}$ & Smart health \\
\hline
\end{tabular}

of natural disaster is the key to save people's life. In [15] [16], authors reported a new type of seismic system, MyShake, creatively applying accelerometer sensor of smart phones to realize earthquake early-warning in urban environments.

- Health Care. Health care plays an important role in people's daily life. In [13] [14], authors, based on data analysis and visualization, implemented the TrackYourTinnitus system to reveal new medical aspects on tinnitus and its treatment. What's more, motivated by the COVID-19 pandemic, CovidSens [28] is used to monitor the COVID-19 propagation via GPS, microphone, and camera.

- Urban Management. Urban management, e.g., traffic management and road maintenance, has a tedious, delayprone process in the smart city domain. In [12], authors utilized the CREAM system to provide a timely response for traffic management. SafeStreet was presented to detect road anomaly for the safe drive of drivers [21].

- Social Networking. Websites and social software, e.g., Facebook and Twitter, comprise huge amounts of data to improve the service quality of the social network. In [17] [18], TrackMaison keeps track of social network service usage of smartphone users through data usage, location, usage frequency and session duration for identifying users' social behaviour.
- Other. The appearance of many crowdsensing applications or platforms poses huge challenges for maximizing the utility of sensing resources. For example, to solve the lack of a unified architecture and the incompatibility of algorithms in existing research, authors presented an operating system, CrowdOS [27].

As a result of our extensive evaluation, we find that MCS technology has been widely applied in various scenarios, e.g., smart environment, smart service, smart transportation, smart health, and smart social, leading to several research directions including function realization, participant selection, task allocation, incentive strategies, data mining and visualization, and privacy protection. In these researches, citizens are the major participant of sensing task for MCS. Meanwhile, all these scenarios belong to the smart city domain. However, it is worth noting that MCS technology is still not extensively applied in the smart agriculture domain and farmers are slao not involved in MCS campaigns. Currently, with the rapid development of the economy, even in underdeveloped countries, the proportion of owning smartphones is almost or at least one in every farmer's family [30]. Moreover, farmers who equip with smart devices will obtain better agricultural data due to participating in the whole agricultural production process. Motivated by the above reasons, Motivated by the above reasons, we hope to utilize MCS to cover the existing agricultural data collection system shortage, i.e., SAGIN. 
In our previous study [31], we just proposed the concept of AMCS and analyzed three benefits, i.e., cheaper cost, superior scalability, and balance data granularity and excellent flexibility, of using MCS technology to obtain agricultural data through comparing with SAGIN. However, our previous work needs to improve further due to the lack of considering the challenges caused by integrating MCS with agriculture and the limitations of AMCS. Thus, in this paper, we will fully explore the application of AMCS towards smart agriculture. Compared to traditional MCS applications that mainly focused on urban areas, AMCS is expected to improve the existing agricultural data collection system and facilitate innovative agriculture development.

\section{A. Contributions \& Organization}

The main contributions of our work are:

(i) Based on the deployed MCS system, we review the typical MCS's applications designed for different urban scenarios. Comparing with existing agricultural data collection method, we further analyze the advantages and disadvantages of applying AMCS in the farming scenario.

(i) We analyze the crucial factors of combining MCS with agriculture, including the number of potential users, developed agriculture-related APPs, farmer's experience, and cooperation between agribusiness and farmers;

(iii) We propose six application scenarios and list future research issues of AMCS.

The rest of the paper is structured as follows. Section II reviews the research status of data collection in smart agriculture and further analyzes the advantages and disadvantages through comparing AMCS with existing methods for collecting agricultural data. Section III examines the crucial factors of combining AMCS with agriculture. We further elaborate on the potential applications and future research issues of AMCS in section IV and section V, respectively. Finally, conclusions are drawn in Section VI.

\section{DATA COLLECTION IN SMART AGRICULTURE}

According to the different factors of production, agricultural industries, i.e., farming, stockbreeding, forestry, aquaculture, and sideline, serve different products with a similar production process. Therefore, in this section, farming is taken as an example to investigate data varieties and the existing data collection system.

\section{A. Data varieties in farming}

The whole process of agricultural production can be regarded as a complete production chain and generate a large amount of data with many types, e.g., humidity [32], spectral data [33], and soil moisture content [34]. Fig. 2 shows that the production chain is divided into three phases: Pre-Production Phase, In-Production Phase, and Post-Production Phase. Furthermore, every stage comprises several production links, e.g., production preparation and farmland management.

1) In Pre-Production Phase, limited preparation time forces farmers to make planting plans quickly, e.g., crop type and area, which enormously rely on the information feedback of market sale last year. More importantly, the schedule at this phase will affect the final harvest of the crop.

2) In In-Production Phase, according to the change of weather, e.g., rainfall, temperature, and humidity, farmers need to dynamically manage crops planted through Agricultural Technology, e.g., irrigation and spraying pesticides, to increase crop yields as much as possible.

3) In Post-Production Phase, harvested crops typically need to go through several steps, e.g., transportation, storage, and sale, until they are entirely consumed or disposed of due to exceeding the shelf life.

\section{B. Existing data collection method}

1) Space-Air-Ground Integrated Network (SAGIN)

The rapidly developed sensing technologies, e.g., remote sensing and wireless sensing, have significantly enriched agricultural information acquisition methods towards a good data collection system - SAGIN. It is used to gain the growth information of crops and improve the accuracy of farmland management, e.g., irrigation, fertilization, and seeding. SAGIN mainly consists of three parts:

- In the space, Remote Sensing Satellites (RSSs) are used to gather agricultural environment information by taking images, in which 3S technology, i.e., Remote Sensing (RS), Geography Information Systems (GIS), and Global Positioning System (GPS), is adopted to acquire data.

- In the air, Unmanned Aerial Vehicles (UAVs), including other vehicles, e.g., agricultural aircraft [35], are applied to gather information of target areas by carrying specific sensors, e.g., hyperspectral camera [36].

- On the ground, Wireless Sensor Networks (WSNs), which consist of deployed wireless sensors with various protocols [37], e.g., Zigbee, Bluetooth, and Lora, can collect data, e.g., temperature and humidity, in the region of interests.

\section{2) Crowdsourcing}

Crowdsourcing(CS) enforces community-oriented application purposes and leveraging extensive user participation for data collection [38]. In developing countries, due to limited resources, current surveillance efforts are unable to provide sufficient data for monitoring disease and pest attacks over a vast geographic area efficiently and effectively. Thus, in [39], authors used CS to offer real-time surveillance data on viral disease and pest incidence and severity. The research aims to eventually use this data to build an automated diagnostic tool for cassava diseases and provide a real-time situation map of the state of illness in the whole country.

\section{Comparison}

In this section, we make an overall comparison with existing data collection methods through the following seven factors, as shown in Table II.

1) Comparing AMCS with existing data collection methods

- Data granularity. Resolution of sensing equipment is a crucial measurement index to data granularity. WSNs, 


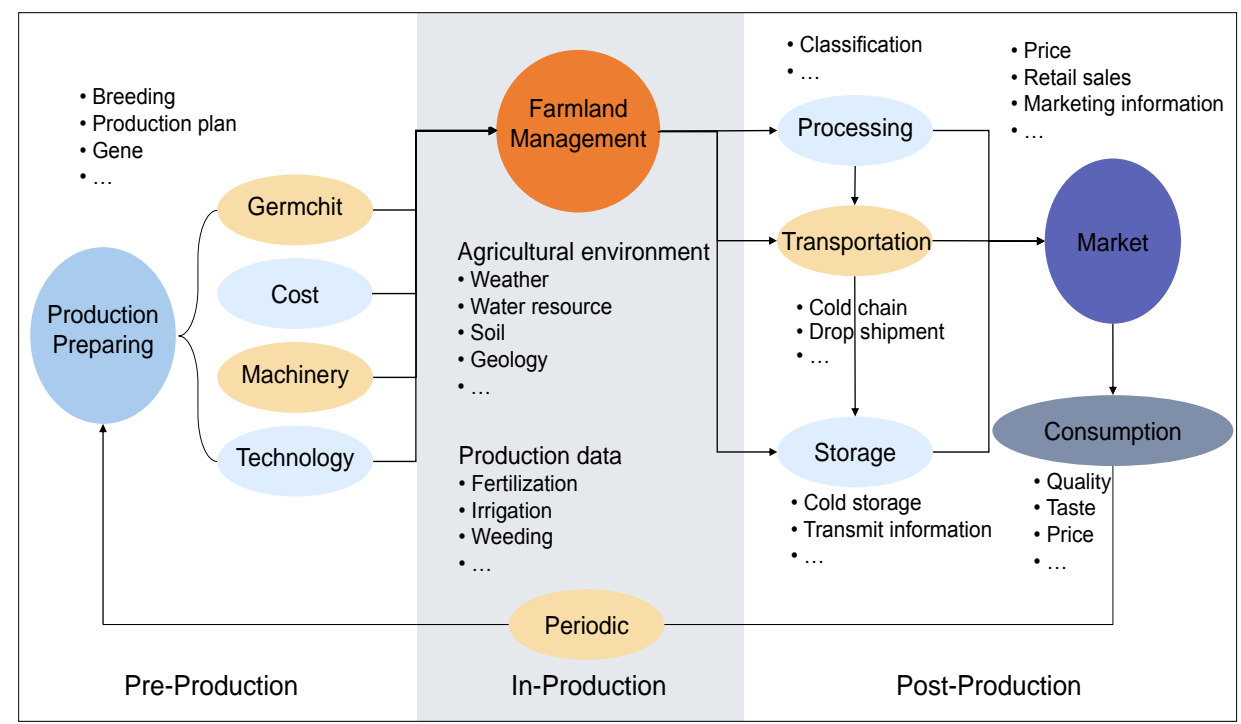

Fig. 1. Data types in agricultural production

TABLE II

COMPARISON OF AMCS WITH SAGIN IN SMART AGRICULTURE.

\begin{tabular}{|c|c|c|c|c|c|}
\hline Item & Space & Air & Ground & CS & AMCS \\
\hline Gathering equipment & RSSs & UAVs & WSNs & $\begin{array}{l}\text { Including but not limited } \\
\text { to smart phones }\end{array}$ & Smart phones \\
\hline Data granularity & Coarse-grain & Coarse-grain & Fine-grain & Fine-grain & Fine-grain \\
\hline $\begin{array}{l}\text { Flexibility (mobility } \\
\text { and expansibility) }\end{array}$ & $\begin{array}{l}\text { Moveable but not be } \\
\text { expanded }\end{array}$ & $\begin{array}{l}\text { Moveable and can be } \\
\text { expanded }\end{array}$ & $\begin{array}{l}\text { Immovable but can be } \\
\text { expanded }\end{array}$ & $\begin{array}{l}\text { Mobility based on event } \\
\text { and can be expanded }\end{array}$ & $\begin{array}{l}\text { Mobility based on loca- } \\
\text { tion and can be expanded }\end{array}$ \\
\hline Data integrity & Full coverage & Full coverage & Full coverage & $\begin{array}{l}\text { Incomplete } \\
\text { coverage }\end{array}$ & $\begin{array}{l}\text { Incomplete } \\
\text { coverage }\end{array}$ \\
\hline Implicit data collection & Unable & Unable & Unable & Available & Available \\
\hline $\begin{array}{l}\text { Cost in deploy, use, } \\
\text { and maintenance }\end{array}$ & $\begin{array}{l}\text { High cost in all three } \\
\text { aspects }\end{array}$ & $\begin{array}{l}\text { High cost in all three } \\
\text { aspects, lower than RSSs }\end{array}$ & $\begin{array}{l}\text { Low cost in use, high } \\
\text { cost in other aspects, }\end{array}$ & $\begin{array}{l}\text { Low cost in all three } \\
\text { aspects }\end{array}$ & $\begin{array}{l}\text { Low cost in all three } \\
\text { aspects }\end{array}$ \\
\hline
\end{tabular}

CSs, and AMCSs can obtain fine-grain data via sensing information in a close range. Comparatively, the resolution of RSSs and UAVs is lower than the above three methods due to acquire data with remote sensing technology in a relatively distant location.

- Flexibility. This category includes mobility and expansibility. RSSs can round with the earth, but they are difficult to extend extra function because they are deployed with a specific purpose, e.g., meteorological satellite. UAVs can be expanded via loading different sensing equipment and quickly fly to appointed areas. Although WSNs can be expanded with the built-in interface, e.g., USB, they cannot move by themselves. CSs and AMCSs have great flexibility through expanding extra functions with the interface of smart devices, e.g., type- $\mathrm{C}$ and reaching appointed areas with the mobility of users.

- Data integrity. In this category, RSSs, UAVs, and WSNs can obtain comprehensive information with remote sensing technology, multiple measurement, and network, respectively. But in a CS or AMCS campaign, not all the tasks can be completed, which will lead to the lack of data in partial areas.

- Data quality. RSSs, UAVs, and WSNs usually adopt professional equipment to collect data, ensuring the quality of collected data. However, affected the subjective behaviour of users, e.g., habits, collecting data with CSs and AMCSs is hard to ensure the data quality.

- Implicit data collection. Both CSs and AMCSs can get unstructured data, e.g., text description of plant planning, in the pre-production phase and the post-production phase, connecting physical and digital worlds and effectuating data sharing in the overall agricultural industry chain.

- Cost. Comparing with RSSs, UAVs, and WSNs, AMCSs complete sensing tasks with equipped smart devices. Hence, it will not take the cost of deployment and maintenance. It is worth noting that crowdsourcers may need to provide extra equipment for users in a CS campaign [39], which will require extra cost compared with AMCSs.

2) Summary 


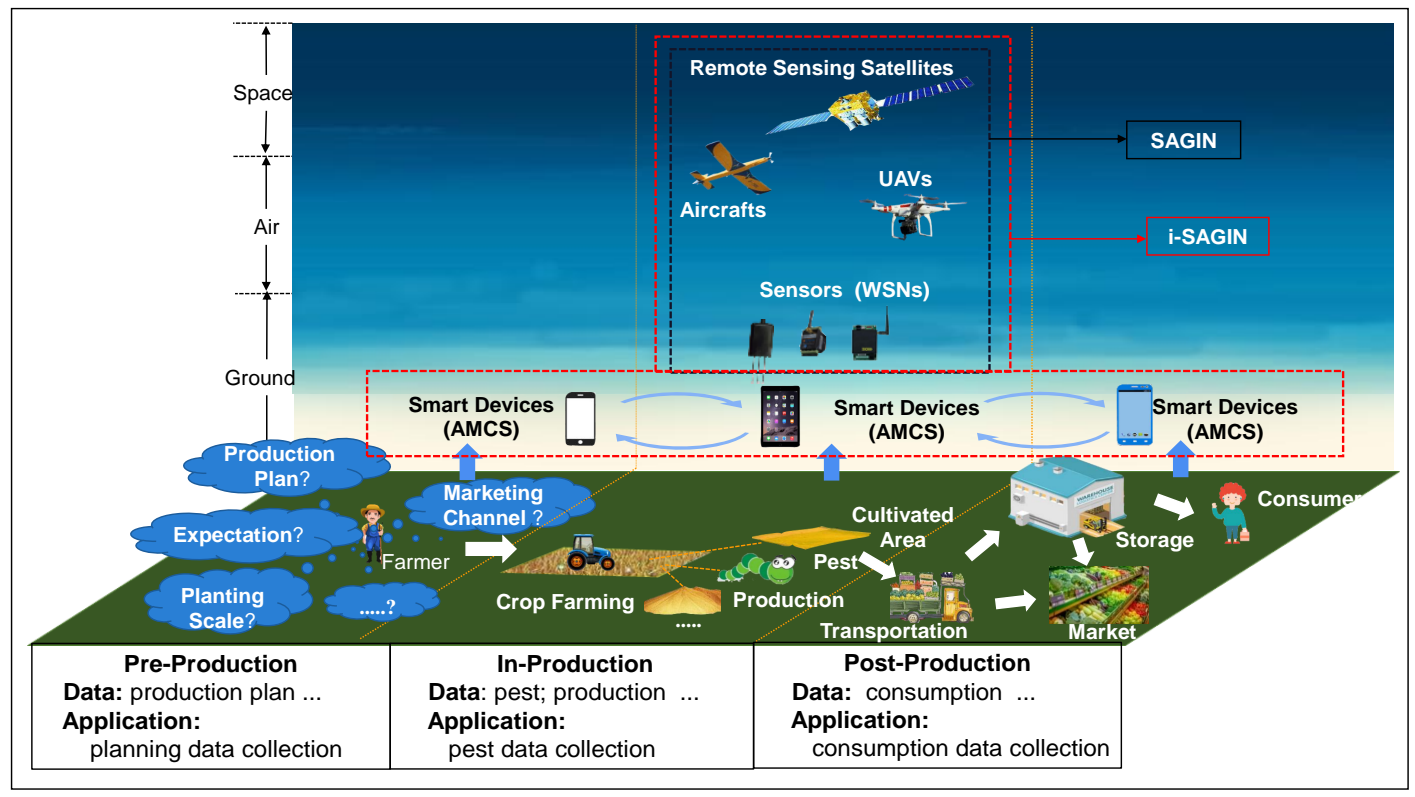

Fig. 2. Integrating AMCS into SAGIN for improving the efficiency of data collection in agricultural production.

Comparing with RSSs, UAVs, and WSNs, AMCSs significantly improved flexibility, collected implicit data, and cost. However, the limitations of AMCSs in data integrity and quality still should be noticed. Thus, AMCS can become an integral approach of acquiring agricultural data for further improving the efficiency of data collection in different agricultural application scenarios rather than wholly replacing the SAGIN system. Besides, comparing with CSs, AMCSs have two advantages: 1) more flexible due to select participants based on location and 2) more convenient participation mode due to use their mobile phones of users to join in sensing tasks. For example, in [39], the crowdsourcer, who is the institution that requests the data, recruited farmers offline artificially for specific crowdsourcing tasks and provided smartphones for the participates. However, AMCS can choose appropriate participants based on real-time location and actual landform for performing multiple tasks. Meanwhile, using farmers' smartphones for data collection can further reduce the cost of tasks.

\section{THE CRUCIAL FACTOR OF COMBINING AMCS WITH AGRICULTURE}

Although there are advantages mentioned above comparing AMCS with the existing collection systems, it is still necessary to analyse how to apply AMCS in agriculture. In this section, the critical factor of combining AMCS with agriculture will be diagnosed with the following fourfold factors, as shown in Fig. 3.

\section{A. Lots of potential users}

According to the latest data published by The World Bank in 2019 [40], especially in developing countries, the ratio of employment in agriculture is generally over $25 \%$ of total jobs, e.g., the highest value was in Burundi: $92.04 \%$. Even in the USA, one of the most developed agricultural countries globally, more than 3 million employees are working in agriculture with the smartphone. Therefore, those employees are potential users for AMCS to collect agricultural data.

\section{B. Developed agriculture-related APPs}

Recently, developed agriculture-related APPs based on android or IOS operating system have been widely used in agricultural production [41], which makes it possible to use the smartphone for collecting varieties of data. For instance, in [42], authors designed an application to analyze brightness with cameras of smartphones, and in [43], mobile phones were used as soil colour sensors. Easy operation and practicability will improve the utilization rate of these APPs, which can generate massive data. Thus, developed APPs are also an indispensable condition for the application of AMCS.

\section{Farmer's experience}

Compared to practitioners of other industries, farmers have more professional knowledge of agriculture, providing more accurate agriculture data. In data collection, farmers' unique experience accumulated from agricultural production may also transfer to meaningful data. For instance, when a novel type of plant disease's photos needs to be collected, farmers, based on their experience, can add the descriptions of the disease to refer to data collectors. Therefore, AMCS can deeply integrate into agriculture with the help of farmers' experience.

\section{Cooperation between agribusiness and farmers}

Agribusinesses that are the primary collectors of agricultural data generally build cooperation with farmers to promote their new products and technologies and increase farmer's income, which is the key to converting farmers to AMCS's users. Based 


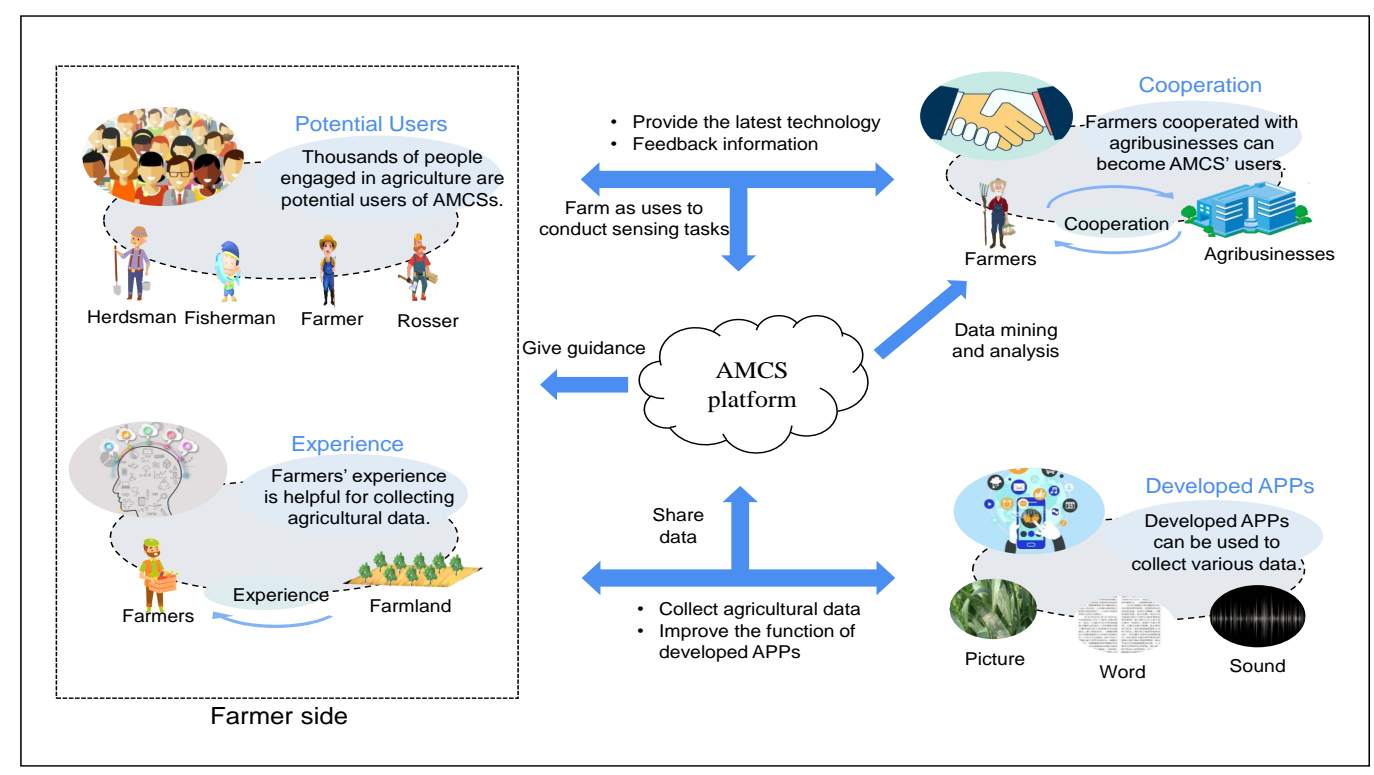

Fig. 3. The crucial factor of combining AMCS with agriculture

on the existing cooperation, farmers will be more enthusiastic about sharing valuable data. Meanwhile, agribusiness will get reliable feedback on their products and technologies. Thus, cooperation between agribusiness and farmers is powerful support for the application of AMCS.

\section{Potential applications of AMCS}

Based on the current states in agricultural production, we propose six potential applications of AMCS, as shown in Fig. 4.

\section{A. Measure cultivated area}

It is significant for the agricultural policy-making sector to predict the area size increasing or decreasing via measuring cultivated area annually. Governments generally use $3 \mathrm{~S}$ technology to measure the cultivated regions of the whole country roughly. The technology has higher accuracy toward largescale farmland but low accuracy for small-scale farmland, e.g., family farm, due to intolerant error and high cost of usage. In this case, farmers with smartphones walking around the edge of the cultivated land can measure the cultivated area size. Of course, the final results need to calibrate subsequently. Combining crops' variety and ultimate yield, the planting area and crop production in the required areas can more accurately obtain.

\section{B. Collect meteorological disaster information}

Agricultural meteorological disasters [44], e.g., floods, frosts, snowstorms, and hail, can reduce the production of crops, leading to severe economic loss to farmers. During the outbreak period of disasters, acquiring detailed emergency information can help the government respond to disaster development situations and provide a reference for the followup compensation of agricultural insurance. Based on these considerations, as eyewitnesses of catastrophe, farmers can obtain more detailed information of the outbreak area, which can improve the quality of the data collected by RSSs.

\section{Collect pest and disease images}

The crop's pests and diseases are one of the crucial factors causing crop production reduction. To get more information about them, researchers have to spend a lot of time and money going to the field to take pest and disease images. Farmers who have experience in identifying pests and diseases can undertake such a task. Hence, researchers can save more time to focus on the analysis of collected data. Besides, many existing android-based APPs of the smartphone, e.g., [45], have been already applied to recognize crop's pests and diseases, which can be combined with MCS technology to collect required images. In this way, apart from extending the data set of the pests and diseases' prints, researchers can study the migration regularity of migratory pests through recorded photos.

\section{Plan for production}

Unmarketable agricultural products cause the waste of farming resources and inevitably lead to financial losses among farmers. Especially in Pre-Production phase, farmers put into the production of the hot-selling products recklessly, resulting in surplus production. Furthermore, in Post-Production Phase, farmers, merchants, and consumers merely possess partial circulation data, e.g., storage data, marketing data, and consumption data, causing unmatched information the supply and demand. Therefore, sharing data among participants during agricultural production is the key to solving this problem of unmarketable agrarian products. 


\section{E. Cooperative sensing with IAM}

Compared to traditional machinery in agriculture, Intelligent Agricultural Machinery (IAM) can increase the operational efficiency of production by installing a variety of wireless sensors, e.g., flow sensors for precision sowing and precision fertilization. Based on these wireless sensors, a lot of meaningful data, e.g., fertilization amount and seeding amount, can be collected, assisting farmers in judging the operational quality of IAM. Specifically, farmers can utilize the computing power of smartphones to visualize the received data collected by wireless sensors. Simultaneously, the collected data will be a historical record and upload to the server for storage. Thus, this application displays the cooperative sensing of IAM and AMCS.

\section{F. Identify the quality of fruits}

With the development of sensing technology, the sensing ability of smartphones become more powerful. For example, Changhong $\mathrm{H} 2$ [46] is the first smartphone embedded with a small-scale molecular spectroscopy sensor, which can obtain fruits' growth paramentes, e.g., sweetness and moisture, by collecting their spectral data. Thus, researchers can study more the quality identification of fruits by analyzing these parameters, which can also help farmers know the growth situation of fruits as early as possible so that they can are capable of dynamically adjust management schemes for maximizing their profits.

\section{Open Research Issues Based on Agricultural CHARACTERISTICS}

Practical applications combined with agricultural and rural characteristics can generate particular research problems in AMCS. In this section, we first summarize the agrarian characteristics. Then, based on these characteristics, we highlight several research issues, as shown in Fig. 5.

\section{A. Agricultural Characteristics}

The characteristics of agriculture determine the difference between AMCS and other MCS paradigms, including:

- Seasonality and regionality. Seasonality reflects that the production environment is changeable, e.g., rainfall in summer and snowfall in winter. Regionality means the differences in production patterns, e.g., mainly planting wheat in the north of China and rice in the south of China.

- Diverse species and multi-type data. Diverse species means a wide range of data types that generate different application scenarios. Multiple types of data represent heterogeneity, which brings challenges to data transmission and visualization as well as data fusion.

- Base station deployed unevenly. Due to the low population density, the base stations deployed in rural areas are sparse and uneven, resulting in insufficient network coverage and unstable connectivity and affecting data transmission. Consequently, the less number of base stations will further cause worse accuracy of GPS positioning.
- Farmers live in villages. Farmers usually live in villages surrounded by farmland, leading to unreachable areas when farmers are not within a busy-farming period since they will not frequently go to their farmlands to work. Therefore, the temporal-spatial distribution of participants in the area of the sensing task is extremely uneven, which cannot guarantee to complete all sensing tasks for obtaining sufficient data.

\section{B. General Research Issues}

- Task allocation mechanism based on multiple constraints. Task quality is one of the important factors for designing task assignments in the MCS [47], e.g., 1) the balance of coverage and cost [48] and 2) task duration [49]. However, in the agricultural scenario, the task assignment of AMCS will meet new constraints, e.g., monitoring the migration trajectory of migratory pests should have sufficient coverage and fine-grained data. Meanwhile, task allocation in AMCS is limited by time due to the seasonality of pest migration. Moreover, multi-types migratory pests mean multiobjective tasks assigned in the same period to improve the work efficiency of AMCS.

- Data transmission strategy based on actual need. Considering the impact of species diversity and unevenly deployed $4 \mathrm{G}$ base stations, using smartphones to transmit data in rural areas will be affected by the following fourfold factors:

1) The different situation of signal strength, including average signal, weak signal, and no signal, may occur during data transmission, consequently affecting the efficiency of data uploading;

2) According to the urgency of the task, data transmission comprises real-time transmission and delay-tolerant transmission [50];

3) Selecting transmission mode, e.g., 4G, 5G or WiFi, also needs to be considered based on the actual situation;

4) Heterogeneous data, e.g., text data, sound data, and image data, can also affect data transmission.

Combinations of these above factors are all possible in practical applications and deserve further research effort.

- Incentive mechanism based on the task's difficulty. The plight of the task includes two aspects: 1) Areas that are farther away from participants and more difficult to reach, e.g., no path to arrive, are equivalent to tasks that are more difficult to complete, requiring task publisher to pay more reward for motivating farmers to participate; 2) Sensing tasks that require more data, i.e., more data types and more data amount, are also considered difficult to complete. Therefore, considering the above twofold factors, dynamic rewards based on the task's difficulty will be beneficial to motivate more farmers to participate in more difficult sensing tasks based on the actual locations of participants.

- Data quality evaluation based on information's priority. Due to the lack of benchmarks, existing researches mainly adopt specific indicators to measure the quality of data in MCS, e.g., the study [51] took context as the indicator, which did not consider the quality of location information, i.e., GPS data. In the agriculture domain, data with an inaccurate location is invalid data, which needs to be calibrated or 


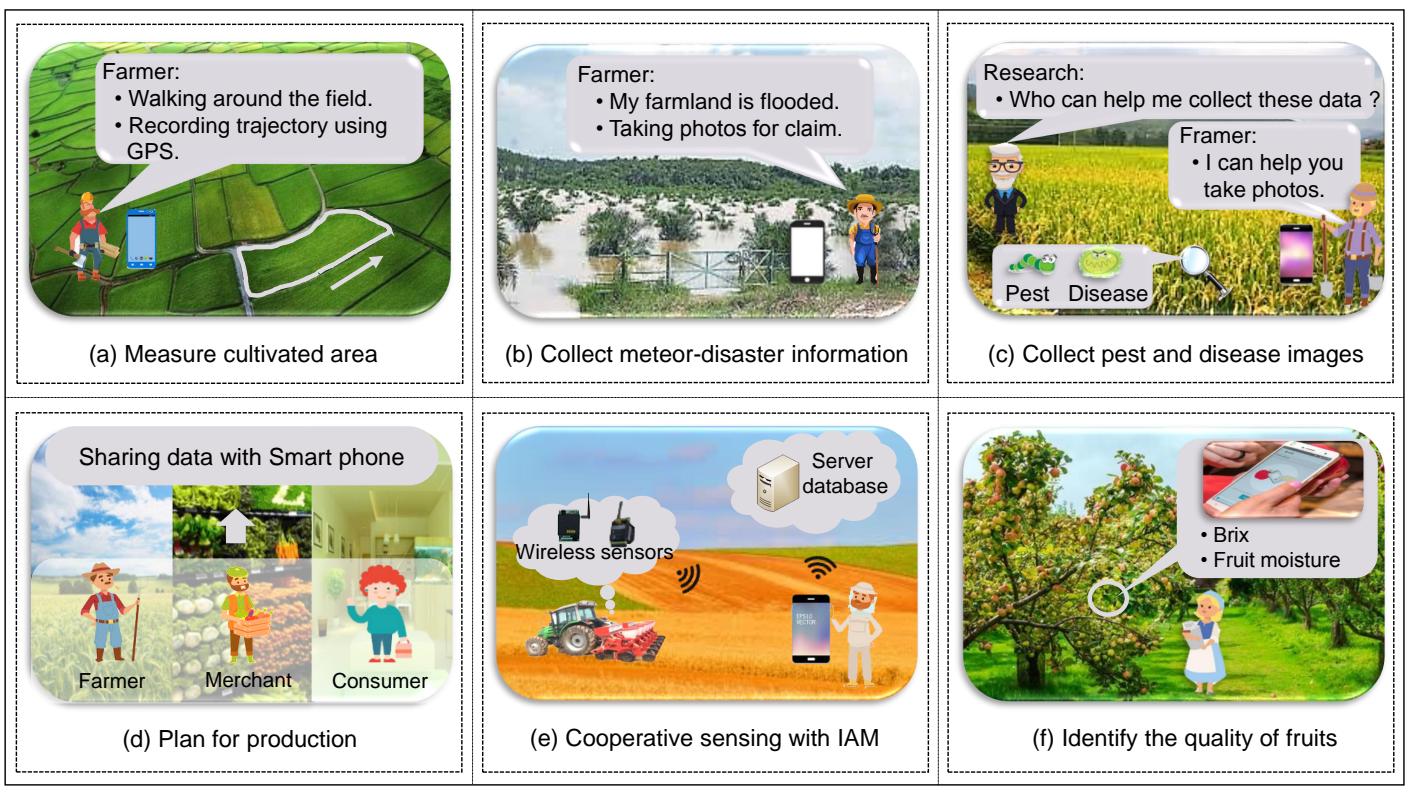

Fig. 4. Six potential applications of AMCS

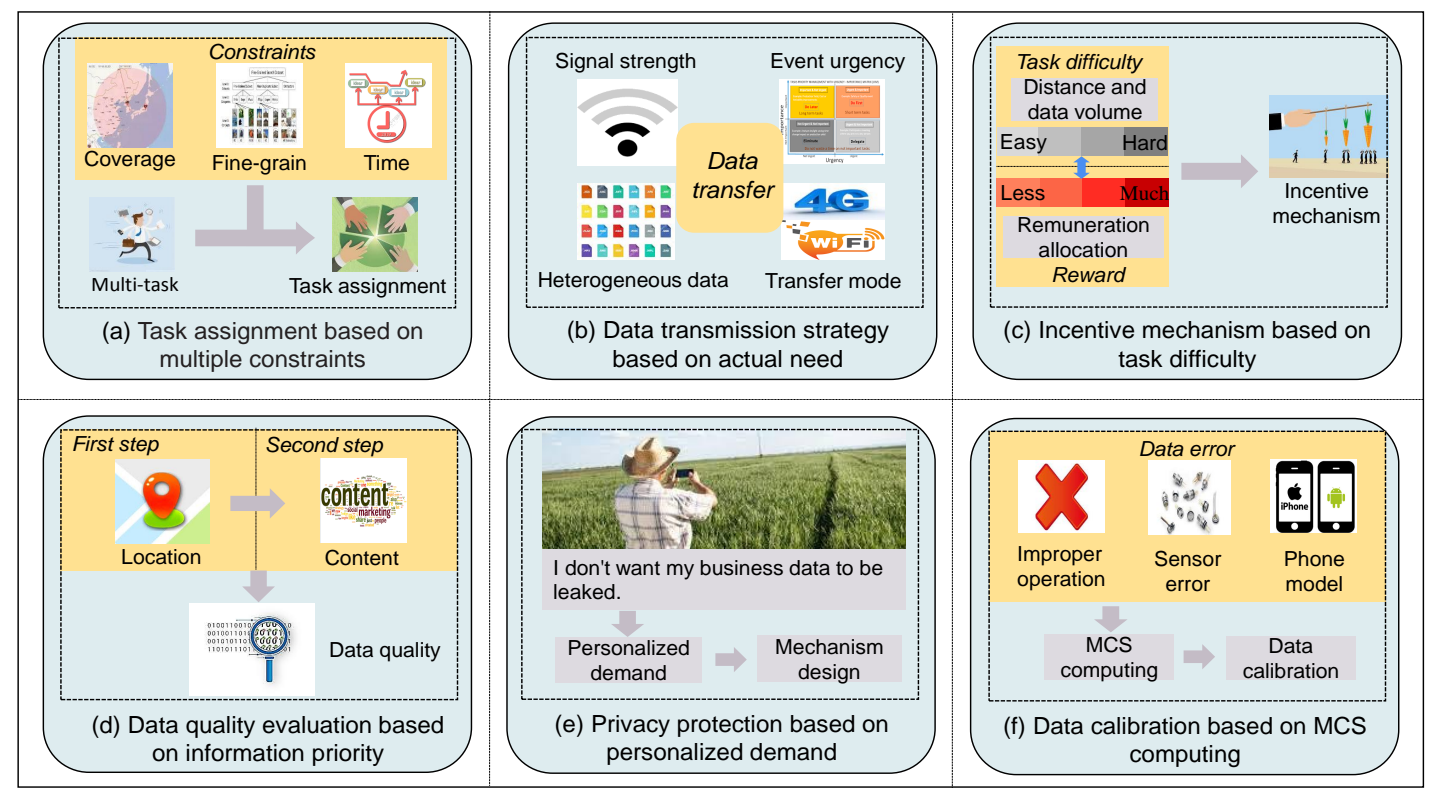

Fig. 5. Open research issues.

cleansed. However, in farmland, manual calibration is also incorrect due to the lack of reference objects. According to existing technologies, integrating GPS and 4G base stations is the current best positioning scheme. Nevertheless, it can be easily affected by insufficient satellite coverage, uneven deployment of $4 \mathrm{G}$ base stations, and uncontrollable jamming signals. Therefore, when participants collect data, the quality of GPS data need to be evaluated because GPS signals are very likely to shift.

- Privacy protection based on personalized demand. Privacy protection varies from MCS [52] [53] with citizens as participants to AMCS with farmers as participants. In rural areas, farmers frequently access their fields to examine the production status of crops as one of the motivations for them to participate in these sensing tasks. In this case, the data collected via farmers is very likely to leak their privacy information, e.g., their income according to the harvest, which will prevent them from participating in these sensing tasks. However, these data may be necessary for certain special sensing tasks. Thus, the privacy protection strategy needs to further optimize in AMCS.

- Data calibration based on MCS computing. Compared with professional equipment, e.g., spectrometer, using smartphones to collect data directly generates more errors, resulting in the collected data being unusable. According to our investigation, the causes of data errors mainly come from 
the following three aspects: 1) caused by the non-standard operation of the user; 2) caused by the sensor accuracy of a smartphone; 3) caused by sensor accuracy difference among different types of smartphones. To ensure the same accuracy as that of traditional equipment, smartphones with powerful computing capacity [41] need to integrate calibration algorithms for improving the quality of collected data.

\section{CONCLUSION AND INSIGHT}

As a promising paradigm, smart agriculture integrates advanced intelligent technologies, e.g., Big Data and the Internet of Things, into agricultural production, leading to many areas of research and applications, e.g., Agricultural Big Data. However, it still has has significant limitations for data collection in terms of cost, scalability, data granularity, and flexibility. In this paper, to solve these problems, we propose to apply MCS in the smart agriculture domain. Through comprehensive analysis, we find AMCS is feasible to collect agricultural data and has significant benefits. The insights of this paper are threefold:

- For farmers, smartphones are not merely the traditional equipment for communication. Nowadays, they can become an essential tool for managing agricultural production and increasing income.

- We update and expand the concept of AMCS for improving its applicability. Meanwhile, by considering the large number of potential users, developed agriculture-related APPs, farmers experience, and cooperation between agribusiness, we have justified the feasibility of AMCS.

- Consequently, we explore the potential applications and the research issues of AMCS for further studies.

\section{ACKNOWLEDGMENT}

This work was supported in part by the National Natural Science Foundation of China under Grant 62072248 and in part by China Scholarship Council (CSC).

\section{REFERENCES}

[1] S. Wolfert, L. Ge, C. Verdouw, and M. J. Bogaardt, "Big data in smart farming: a review," Agricultural Systems, Vol. 153, pp. 69-80, Feb. 2017.

[2] J. Hammond, S. Fraval, J. Van Etten, J. G. Suchini, L. Mercado, T. Pagella, and V. D. albuena, "The rural household multi-indicator survey for rapid characterisation of households to inform climate smart agriculture interventions: description and applications in east africa and central america." Agricultural Systems, Vol. 151, pp. 225-233, 2017.

[3] J. Liu, Y. Shi, Z. D. Fadlullah, and N. Kato, "Space-Air-Ground integrated network: a survey," IEEE Communications Surveys \& Tutorials, Vol. 20, No. 4, pp. 2714-2741, May. 2018.

[4] H. Ma, D. Zhao, and P. Yuan, "Opportunities in mobile crowd sensing," IEEE Communications Magazine, Vol. 52, No. 8, pp. 29-35, Aug. 2014.

[5] R K. Ganti, F. Ye, and H. Lei, "Mobile crowd sensing: current state and future challenges," IEEE Communications Magazine, Vol. 49, No. 11, pp. 32-39, Nov. 2011

[6] L. Wang, D. Zhang, Y. Wang, C. Chen, X. Han, and A. M'hamed, "Sparse mobile crowdsensing: challenges and opportunities." IEEE Communications Magazine, Vol.54 No. 7, pp. 161-167, 2016.

[7] Z. Zhou, H. Liao, B. Gu, K. M. S. Huq, S. Mumtaz, and J. Rodriguez, "Robust mobile crowd sensing: when deep learning meets edge computing.” IEEE Network, Vol. 32, No. 4, pp. 54-60, 2018.

[8] D. Zhang, H. Xiong, and L. Wang, "CrowdRecruiter: selecting participants for piggyback crowdsensing under probabilistic coverage constraint," Proc. 2014 Acm International Joint Conference on Pervasive and Ubiquitous Computing, pp. 703-714.
[9] J. Wang, Y. Wang, and D. Zhang, "Real-time and generic queue time estimation based on mobile crowdsensing," Frontiers of Computer Science, Vol. 11, No. 1, pp. 49-60, 2017.

[10] W. Zamora, E. Vera, and C. Calafate, "GRC-Sensing: an architecture to measure acoustic pollution based on crowdsensing," Sensors, Vol. 18, No. 8, 2018

[11] X Zhao, N Wanga, and R Han, "Urban infrastructure safety system based on mobile crowdsensing," International Journal of Disaster Risk Reduction, Vol. 27, pp. 427-438, 2018

[12] X. Wang, Z. Ning, and X. Hu, "A city-wide real-time traffic management system: enabling crowdsensing in social internet of vehicles," IEEE Communications Magazine, Vol. 56, No. 9, pp. 19-25, Sept. 2018,

[13] R. Pryss, M. Reichert, and B. Langguth, "Mobile crowd sensing services for tinnitus assessment, therapy, and research," Proc. 2015 IEEE International Conference on Mobile Services, pp. 352-359.

[14] R. Pryss, W. Schlee, and B. Hoppenstedt, "Applying machine learning to daily-life data from the TrackYourTinnitus mobile health crowdsensing platform to predict the mobile operating system used with high accuracy: longitudinal observational study," Journal of Medical Internet Research, Vol. 22, No. 6, 2020.

[15] Q. Kong, R M. Allen, and L. Schreier, "MyShake: a smartphone seismic network for earthquake early warning and beyond,' Science Advances, Vol. 2, No. 2, Feb. 2016.

[16] Q. Kong, A. Inbal, and R. M. Allen, "Machine learning aspects of the MyShake global smartphone seismic network" Seismological Research Letters, Vol. 90, No. 2A, PP. 546-552, 2019.

[17] F. Anjomshoa, M. Catalfamo, and D. Hecker, "Mobile behaviometric framework for sociability assessment and identification of smartphone users," Proc. 2016 IEEE Symposium on Computers and Communication, pp. 1084-1089.

[18] Z. Rauen, F. Anjomshoa, B. Kantarci, "Empowering human-computer interaction in securing smartphone sensing," Proc. 2018 IEEE 23rd International Workshop on Computer Aided Modeling and Design of Communication Links and Networks (CAMAD), pp. 1-6.

[19] Q. Xu, R. Zheng, "Mobibee: A mobile treasure hunt game for locationdependent fingerprint collection," Proc. 2016 ACM International Joint Conference on Pervasive and Ubiquitous Computing: Adjunct, pp.14721477.

[20] Q. Xu, R. Zheng, and E. Tahoun, "Detecting location fraud in indoor mobile crowdsensing," Proc. 2017 the First ACM Workshop on Mobile Crowdsensing Systems and Applications, pp. 44-49.

[21] V. Singh, D. Chander, and U. Chhaparia, "SafeStreet: an automated road anomaly detection and early-warning system using mobile crowdsensing," Proc. 2018 10th International Conference on Communication Systems \& Networks (COMSNETS), pp. 549-552.

[22] D. Bonino, M. T. D. Alizo, and C. Pastrone, "WasteApp: smarter waste recycling for smart citizens," Proc. 2016 International Multidisciplinary Conference on Computer and Energy Science (SpliTech), pp. 1-6.

[23] S. Rosen, S. Lee, and J. Lee, "MCNet: crowdsourcing wireless performance measurements through the eyes of mobile devices," IEEE Communications Magazine, Vol. 52, No. 10, pp. 86-91, 2014.

[24] F. Montori, L. Bedogni, and A. Di Chiappari, "SenSquare: a mobile crowdsensing architecture for smart cities," Proc. 2016 IEEE 3rd World Forum on Internet of Things (WF-IoT), pp. 536-541.

[25] F. Montori, L. Bedogni, and L.Bononi, "A collaborative internet of things architecture for smart cities and environmental monitoring," IEEE Internet of Things Journal, Vol.5, No. 2, pp. 592-605, 2017.

[26] F. Montori, L. Bedogni, and G. Iselli, "Delivering iot smart services through collective awareness, mobile crowdsensing and open data," Prof. 2020 IEEE International Conference on Pervasive Computing and Communications Workshops (PerCom Workshops), pp. 1-6.

[27] Y. Liu, Z. Yu, and B. Guo, "CrowdOS: a ubiquitous operating system for crowdsourcing and mobile crowd sensing," IEEE Transactions on Mobile Computing, 2020. (Early Access)

[28] M. T. Rashid, D. Wang, "CovidSens: a vision on reliable social sensing for COVID-19," Artificial Intelligence Review, Vol. 54, No. 1, pp. 1-25, 2021.

[29] G. Yang, S. He, Z. Shi, and J. Chen, "Promoting cooperation by the social incentive mechanism in mobile crowdsensing," IEEE Communications Magazine, Vol. 55, No. 3, pp. 86-92, 2017.

[30] O. A. Ogbeide and I. Ele, "Smallholder farmers and mobile phone technology in Sub-Sahara Agriculture," Mayfair Journal of Information and Technology Management in Agriculture, Vol. 1, No. 1, pp. 1-19, 2015.

[31] Y. Sun, W. Ding, L. Shu, K. Huang, K. Li, Y. Zhang, and Z. Huo, "Poster: when mobile crowd sensing meets smart agriculture," Proc. 2019 Acm Turing Celebration Conference-China, No. 49. 
[32] S. R. Prathibha, A. Hongal, and M. P. Jyothi, "IoT based monitoring system in smart agriculture," Proc. 2017 International Conference on Recent Advances in Electronics and Communication Technology (ICRAECT), pp. 81-84, March. 2017.

[33] L. Ravikanth, D. S. Jayas, N. D. White, P. G. Fields, and D. W. Sun, "Extraction of spectral information from hyperspectral data and application of hyperspectral imaging for food and agricultural products," Food and Bioprocess Technology, Vol. 10, No. 1, pp. 1-33, 2017.

[34] D. D. Alexakis, F. D. K. Mexis, A. E. K. Vozinaki, I. N. Daliakopoulos, and I. K.Tsanis, "Soil moisture content estimation based on sentinel1 and auxiliary earth observation products: a hydrological approach," Sensors, Vol. 17, No. 6, pp. 1455, 2017.

[35] E. R. Hunt Jr and C. S. Daughtry, "What good are unmanned aircraft systems for agricultural remote sensing and precision agriculture?" International Journal of Remote Sensing, Vol. 39, No. 15, pp. 53455376, 2018

[36] R. Nsi, E. Honkavaara, M. Blomqvist, P. Lyytikinen-Saarenmaa, T. Hakala, N. Viljanen, and M. Holopainen, "Remote sensing of bark beetle damage in urban forests at individual tree level using a novel hyperspectral camera from UAV and aircraft," Urban Forestry and Urban Greening, Vol. 30, pp. 72-83, 2018.

[37] D. Shinghal and N. Srivastava,"Wireless sensor networks in agriculture: for potato farming," Neelam, Wireless Sensor Networks in Agriculture: For Potato Farming, Sept, 2017.

[38] A. Ghezzi, D. Gabelloni, A. Martini, and A. Natalicchio, "Crowdsourcing: a review and suggestions for future research," International Journal of Management Reviews, Vol. 20, No. 2, pp. 343-363, 2018.

[39] D. Mutembesa, C. Omongo, and E. Mwebaze, "Crowdsourcing realtime viral disease and pest information: A case of nation-wide cassava disease surveillance in a developing country," Proc. 2018 Sixth AAAI Conference on Human Computation and Crowdsourcing, June. 2018.

[40] "Employment in agriculture country rankings", https://www.theglobaleconomy.com/rankings/, accessed: 2020-0123.

[41] S. Pongnumkul, P. Chaovalit, and N. Surasvadi, "Applications of smartphone-based sensors in agriculture: a systematic review of research," Journal of Sensors, 2015.

[42] S. Sumriddetchkajorn, "How optics and photonics is simply applied in agriculture?" Proc. 2013 International Conference on Photonics Solutions, Vol. 8883, June. 2013.

[43] L. Gomez-Robledo, N. L opez-Ruiz, M. Melgosa, A. J. Palma, and L. F. Capitan-Vallvey, "Using the mobile phone as munsell soil-colour sensor: an experiment under controlled illumination conditions," Computers and Electronics in Agriculture, Vol. 99, pp. 200-208, 2013.

[44] X. Yu, X. Yu, and Y. Lu, "Evaluation of an agricultural meteorological disaster based on multiple criterion decision making and evolutionary algorithm," International Journal of Environmental Research and Public Health, Vol. 15, No. 4, pp. 612, 2018.

[45] Q. Yao, C. Zhang, Z Wang, B. Yang, and J. Tang, "Design and experiment of agricultural diseases and pest image collection and diagnosis system with distributed and mobile device," Transactions of the Chinese Society of Agricultural Engineering, Vol. 33, pp. 184-191, 2017.

[46] "Phone with 'infrared vision' knows how fat you are (hands-on)," https://www.cnet.com/reviews/changhong-h2-preview/, accessed: 201701-06.

[47] W. Gong, B. Zhang, C. Li, “Task assignment in mobile crowdsensing: Present and future directions," IEEE Network, Vol. 32, No. 4, pp. 100107, 2018.

[48] H. Xiong, D. Zhang, G. Chen, L. Wang, V. Gauthier, and L. Barnes, "iCrowd: Near-optimal task allocation for piggyback crowdsensing," IEEE Transactions on Mobile Computing, Vol. 15, No. 8, pp. 20102022, 2015.

[49] M. Xiao, J. Wu, L. Huang, R. Cheng, and Y. Wang, "Online task assignment for crowdsensing in predictable mobile social networks," IEEE Transactions on Mobile Computing, Vol. 16, No. 8, pp. 23062320, 2016.

[50] B. Guo, Y. Liu, W. Wu, Z. Yu, and Q. Han, "Activecrowd: A framework for optimized multitask allocation in mobile crowdsensing systems", IEEE Transactions on Human-Machine Systems, Vol. 47, No. 3, pp. 392-403, 2016.

[51] S. Liu, Z. Zheng, F. Wu, S. Tang, and G. Chen, "Context-aware data quality estimation in mobile crowdsensing," Proc. 2017 Conference on Computer Communications, pp. 1-9.

[52] D. He, S. Chan, and M. Guizani, "User privacy and data trustworthiness in mobile crowd sensing," IEEE Wireless Communications, Vol. 22, No. 1, pp. 28-34, 2015.
[53] M. Pouryazdan and B. Kantarci, "The smart citizen factor in trustworthy smart city crowdsensing," IT Professional, Vol. 18, No. 4, pp. 26-33, 2016.

[54] B. Guo, Z. Wang, Z. Yu, Y. Wang, N. Yen, R. Huang, and X. Zhou, "Mobile crowd sensing and computing: The review of an emerging humanpowered sensing paradigm," Proc. 2015 ACM Computing Surveys, pp.

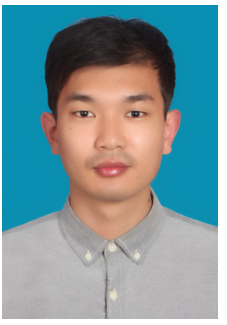

Yuanhao Sun (yhsun.nau@outlook.com) received the B.S. degree in automation from Nanjing Agricultural University, Nanjing, China, in 2016, and the M.S. degree in agricultural engineering from Nanjing Agricultural University, Nanjing, China, in 2018, respectively. e is currently working toward the Ph.D. degree with the College of Engineering, Nanjing Agricultural University, China. His research interest include mobile crowd sensing and optimization algorithm.

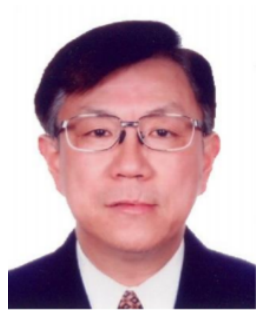

Weimin Ding (wmding@njau.edu.cn) received the B.S. degree from Anhui Agricultural University, Hefei, China in 1981, the M.S. degree from Nanjing Agricultural University, Nanjing, China in 1984, and the Ph.D. degrees from Nanjing Agricultural University, Nanjing, China in 1999. He is the director of Key Laboratory of Intelligent Agricultural Equipment of Jiangsu Province and is currently a Professor at Nanjing Agricultural University, Nanjing, China. His research interests include smart agriculture and facility agriculture.

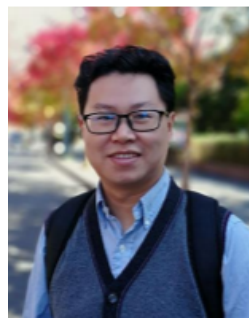

Lei Shu (lei.shu@njau.edu.cn) received the B.S. degree in computer science from South Central University for Nationalities, China, in 2002, the M.S. degree in computer engineering from KyungHee University, South Korea, in 2005, and the Ph.D. degree from the Digital Enterprise Research Institute, National University of Ireland, Galway, Ireland, in 2010. Until 2012, he was a Specially Assigned Researcher with the Department of Multimedia Engineering, Graduate School of Information Science and Technology, Osaka University, Japan. He is currently a Distinguished Professor with Nanjing Agricultural University, China, and a Lincoln Professor with the University of Lincoln, U.K. He is also the Director of the NAU-Lincoln Joint Research Center of Intelligent Engineering. His current research interests include wireless sensor networks and Internet of Things. 


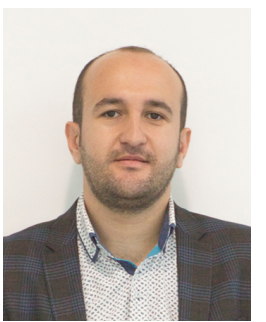

Edmond Nurellari (enurellari@lincoln.ac.uk) was awarded the Carter Prize for the best Ph. D. thesis, titled "Distributed Detection and Estimation in Wireless Sensor Networks: Resource Allocations, Fusion Rules, and Network Security", in the School in the year 2017-18, University of Leeds, UK. Since April 2017, Dr. Nurellari has been a faculty member with the School of Engineering at the University of Lincoln, United Kingdom, where he is currently a Senior Lecturer/Programme Leader in Electrical Engineering/Robotics. His research interests includes machine learning, robotics for communications, distributed signal processing, signal processing on graphs, resource allocations and distributed decisions in WSNs. He has served as an Invited Reviewer for the IEEE Trans. on Signal and Info. Process. over Networks, IEEE Communication Letter Springers Wireless Networks Journal, Springers Digital Signal Processing Journal and IEEE Flagship conferences. Over the past few years, Dr. Nurellari has served as a Guest Associate Editor for the Aerial and Space Networks Section of Frontiers in Space Technologies, Guest Editor of Special Issue "Smart Agricultural Applications with Internet of Things" for Sensors Journal, Editorial Board for Frontiers in Communications and Networks Journal, Journal Topics Board for Journal of Sensor and Actuator Networks, TPC Member for IEEE iSES, International Conference on Smart and Sustainable Agriculture ((SSS'2021)), and a Reviewer for several UKRI grants including EPSRC and Future Leaders Fellowship.

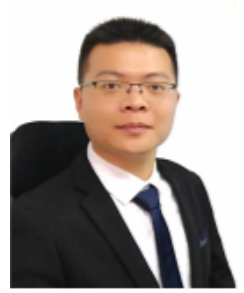

Kailiang Li (kailiang_li@njau.edu.cn) received the B.S. degrees from Guangdong University of Petrochemical Technology, Maoming, China in 2015. $\mathrm{He}$ is currently a Research Assistant at College of Engineering, Nanjing Agricultural University. His research interests are Wireless Sensor Networks and Internet of Things. He has published 11 papers in related conferences, journals in the areas of sensor networks and Internet of Things.

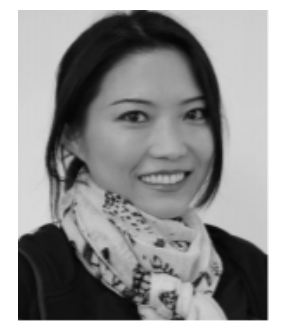

Yu Zhang (Y.Zhang@lboro.ac.uk) received Ph.D degree from the Department of Civil Engineering, University of Nottingham, Nottingham, U.K. in 2011. She is currently a Senior Lecturer in Digital Engineering, the Department of Aeronautical and Automotive Engineering, Loughborough University, U.K. Her research interests include Data Analysis and Machine Learning.

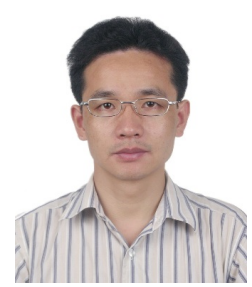

Zhangbing Zhou (zbzhou@cugb.edu.cn) received the B.S. degree from China University Of Geosciences, Wuhan, China, in 1995, and M.S. degree from Institute of Automation, Chinese Academy of Sciences, China, in 2000, and the Ph.D. degree from the Digital Enterprise Research Institute, National University of Ireland, Galway, Ireland, in 2010 . He is a professor at China University of Geosciences (Beijing), China, and an adjunct professor at TELECOM SudParis, France. His research interests include loT sensing networks and services computing.

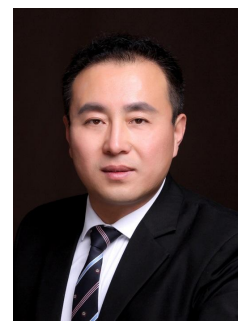

Guangjie Han (hanguangjie@gmail.com)is currently a Professor with the Department of Internet of Things Engineering, Hohai University, Changzhou, China. He received his Ph.D. degree from Northeastern University, Shenyang, China, in 2004. In February 2008, he finished his work as a Postdoctoral Researcher with the Department of Computer Science, Chonnam National University, Gwangju, Korea. From October 2010 to October 2011, he was a Visiting Research Scholar with Osaka University, Suita, Japan. From January 2017 to February 2017, he was a Visiting Professor with City University of Hong Kong, China. His current research interests include Internet of Things, Industrial Internet, Machine Learning and Artificial Intelligence, Mobile Computing, Security and Privacy. Dr. Han is a Fellow of the UK Institution of Engineering and Technology (FIET) and a Senior Member of IEEE. 